\title{
Study on Search and Rescue System for Military and Civil use by using COSPAS SARSAT and Terrestrial Communication Network
}

\author{
Sanguk Lee ${ }^{1}$, Incheol Jeong1, Woo-Geun Ahn' \\ Satellite Research Department, ETRI ${ }^{1}$, Agency for Defense Development ${ }^{2}$
}

${ }^{1}$ Satellite Research Department, ETRI, Daejeon 34129, Korea

${ }^{2}$ Agency for Defence Development Daejeon, Korea

\begin{abstract}
We propose search and rescue system for the both military and civil uses with minimizing burden to COSPAS SARSAT by using terrestrial communication network like LTE as an example in the paper. The terrestrial network may not be limited to LTE but extended to 5G, SigFox, LoRa, etc, later on. For security, special encryption mechanism is applied to the both COSPAS SARSAT and Terrestrial links. Also, operation concept for critical and normal situation. Implementation result and enhancing positioning accuracy by using rescue signal itself from ground signal processing is presented too.
\end{abstract}

\section{Introduction}

COSPAS SARSAT is very famous and popular system and cooperation organization for rescuing people from distress. Tens of thousands of people has been rescued by the system since it started service in 1979. In critical situation like war or normal situation like peace, if military forces and air, terrestrial and maritime military equipment like armoury vehicle, fighter and cargo aircraft, vessels are in trouble like distress or lost, we may need special search and rescue system. In the operational sense, if distress beacon is allowed to personal use, then limited number of simultaneous burst signals some support communication links such as satellite communication link like thuraya, orbcomm, GMPCS and so on or ground links like TRS and LTE. We determined the LTE as assisting communication link and did not choose satellite links due to highly expensive costs to use. In the sense of security, we employed cryptography for guarantee user security. Cosmitscheskaja Sistema Poiska Awarinitsch Sudow (Russian: space system for search of vessels in distress) Search and Rescue Satellite-Aided Tracking (COSPAS SARSAT) system allows utilization of national use field for each member states as described in COSPAS SARSAT(2017). But it has limitation on number of distress signals that can be accommodated at the same time as the limited channel allocation. The burden due to the limitation may be mitigated by additional encrypted satellite link and/or terrestrial link.

\section{COSPAS SARSAT}

The COSPAS-SARSAT system, which is an international search and rescue system, can be divided into distress beacon, satellite system, and ground facilities. Satellite systems have three different system such as Low Earth
Orbit SAR(LEOSAR) and Geostationary Earth Orbit SAR(GEOSAR) as the first generation and Medium Earth Orbit SAR(MEOSAR) as the second generation[1]. The ground facilities are consisted of Local User Terminal, Mission Control Center, and Rescue Control Center). Automatically or manually search and rescue beacon in the event of a vessel / airplane or personal distress as shown in Fig. 1.

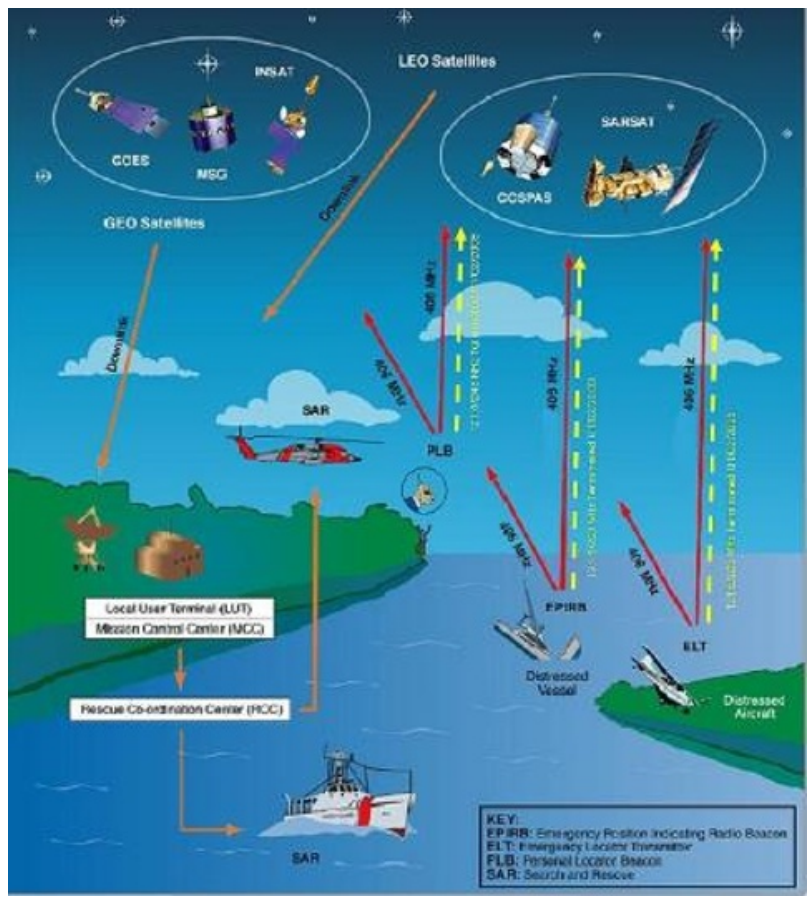

Fig. 1. COSPAS SARSAT configuration and operation

When a distress alert signal on search and rescue beacon is busted out to satellites, it is received by the satellite and relayed to the LUT of the ground system. The LUT

Corresponding author: slee@ etri.re.kr 
computes or acquires the position of search and rescue beacon and transmits the information on the result to the RCC (Rescue Control Center) through the MCC to provide information necessary for expanding the area search and rescue activities. The search and rescue beacon is mounted on a moving object such as a ship or an aircraft including a personal portable device for military and civil uses, and automatically or manually transmits an alarm signal using a wireless communication links when an individual, a ship or an aircraft is in distress or missing, It is a device to help identify a distress situation including localization. .

GPS such as GNSS tells users location to themselves but COSPAS SARSAT such as search and rescue system tells someone else users' location and urgent situation for fast rescue activity possible.

The good news to COSPAS SARSAT is that GNSS system like GPS, GLONASS, Galileo will equip COSPAS SARSAT transponders to their system. Especially, Galileo system already equipped the transponder including return link service which is very good tool for providing the fact that distress signal is acknowledged to distressed person and for control search and rescue beacon to prevent over loading distress signals after it is acknowledged when mass disaster situation.

\section{Military \& Civil Search and Rescue System}

\subsection{Top-level Requirements}

The Military \& Civil Search and Rescue System that is proposed in this paper may be harmonized between military and civil uses. Most of countries employ the COSPAS SARSAT as military use and some counties use dedicated satellite system for the purpose [2]. For the harmonization of them, we made some top level requirements as follows;

- Functional requirements

- Military and civil services should be supported simultaneously but mechanical requirement may be comply application areas and their purposes

- The services starts from the first generation COSPAS SARSAT based and should be expanded to the second generation such as Galileo/SAR, GPS/SAR, and GLONASS/SAR

- Encryption mechanism should be applied for the security and privacy

- Two-way link(or return rink) service should be provided for relieving distressed people and reducing false alerts and increasing capacity of the search and rescue system by burst distress signal control after their recognition.

- Distress signal should be transmitted automatically, manually, and by interrogate signal.
- The alternative communication link(s) should be available to prevent distress signal loss due to tremendous distress signals in mass disaster or critical situation like war.

- Performance requirements

- Most performance requirements on communication links including data link should be comply those of COSPAS SARSAT.

- Positioning accuracies are $50 \mathrm{~m}$ or better with GNSS signal and $1 \mathrm{~km}$ or better without GNSS signal as generic performance

- Recognition and localization of distressed people or equipment should be done 5 minutes in the defined service area.

\subsection{Operation Concept}

A threat level and burden to COSPAS SARSAT in the sense of limitation on channel allocation and assigned frequency band. The operation concept for military and civil search and rescue system is that LTE link is primary for minimizing burden to COSPAS SATSAT and taking benefit (large capacity) of LTE(or PS-LTE) in less threat or peace but COSPAS SARSAT is primary for taking stability of satellite communication of COSPAS SARSAT and preventing vulnerability of LTE that is weak from ground attack in critical situation like a war[3]. LTE(or PS-LTE) is used as distress signal and its return link and COSPAS SARSAT link is used when terrestrial LTE link is not available after pre-defined number of watch-dog timer counts without any acknowledgement from MCC in less threat or peace. For a critical situation like a war, COSPAS SARSAT link may be primary link and its return link and terrestrial LTE link will be the secondary link and its return link when return link does not provide acknowledgement from MCC of COSPAS SARSAT. The terrestrial link is not limited to LTE or PSLTE but can be extended 5G, SigFox, LoRa, NB-IOT, etc. Especially terrestrial IOT network is very useful for the alternatives for the ground cost effective link.

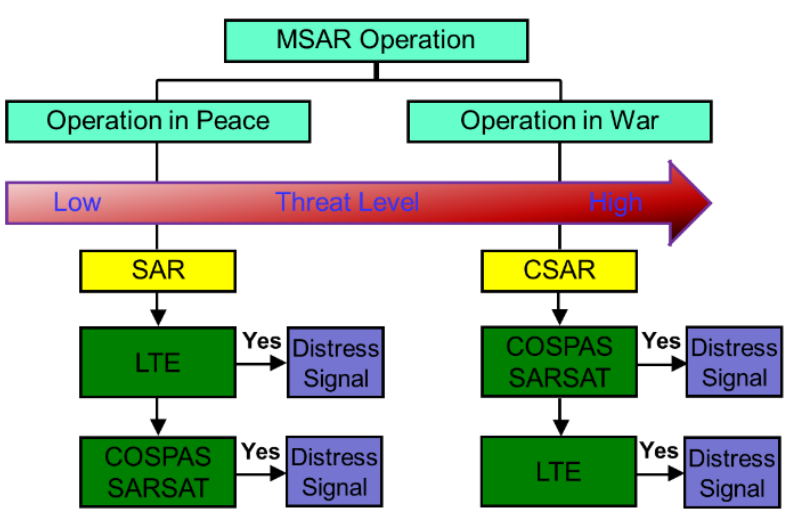

Fig. 2. Operation Concept of military and civil SAR

\section{Military \& Civil Search and Rescue System Prototype and Its Results}




\subsection{SW Modelling \& Simulation Tool}

Modelling and Simulation (M\&S) tool for prototyping of Military \& Civil Search and Rescue System has been developed and its feasibility was checked. Initially, prototyping was made in full software as shown in Fig. 3. We take into account GPS jamming which gives impact on positioning of search and rescue beacon that is equipped with GPS chipset in it. This situation activated positioning of people in distress in alternative way by using distress beacon signal itself.

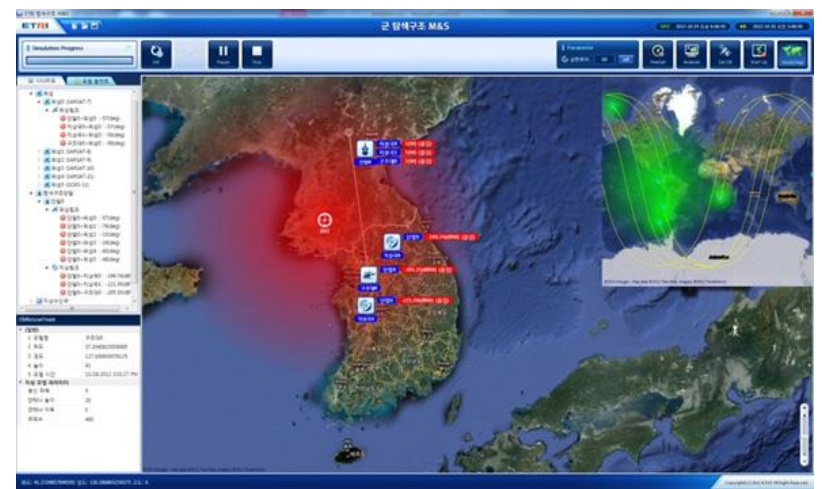

Fig. 3. SW M\&S tool Military \& Civil Search and Rescue System[3].

\subsubsection{Cross Ambiguity Function}

Even though positioning accuracy is not accurate as much as GPS, it gives reasonable accuracy without GNSS signal like GNSS jamming situation. A cross ambiguity function (CAF) method was used to localize the distress signal sources. This methodology provide locations of multiple distress signal sources at the same time by post processing.

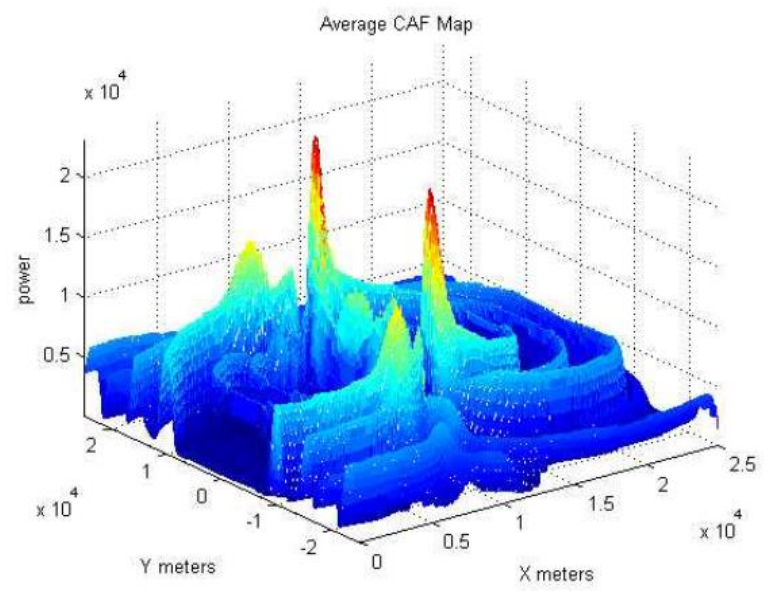

Fig. 4. Localization of distress signal sources by CAF.

\subsubsection{Multi- CAF}

In order to improve localization accuracy, multiple CAF method was used. It provided positioning accuracy about $1 \mathrm{~km}$.

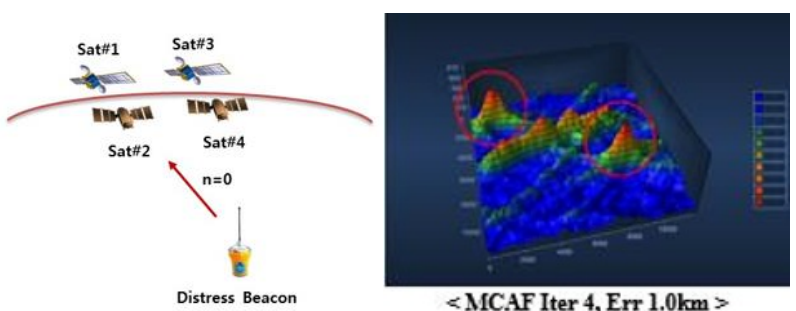

Fig. 5. Localization of distress signal sources by Multi-CAF.

\subsection{Hybrid Modelling \& Simulation Tool}

An LTE communication link were replaced with hardware module as LTE modem with COSPAS SARSAT in SW emulation in data level. It has been merged into hybrid system to check its feasibility with more realistic environment as shown in Fig. 6.

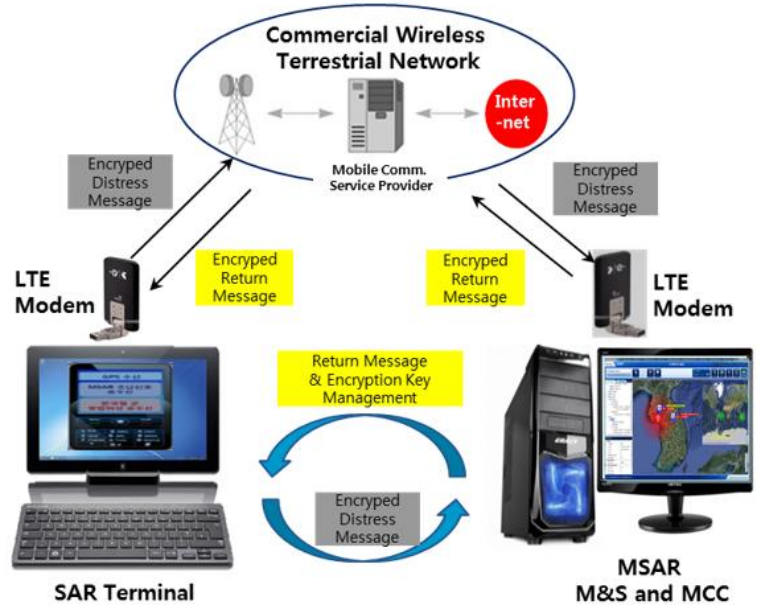

Fig. 6. Configuration of hybrid modelling and simulation tool for Military \& Civil Search and Rescue System

For providing security on the user information including location, encryption scheme was employed to the both LTE and COSPAS SARSAT message protocols. Encryption by 128 bits AES/CFB \& Key management[3][4][5][6] was utilized for COSPAS SARSAT messages by using National User Protocol message format and data fields as shown in figures 7 and 8 .

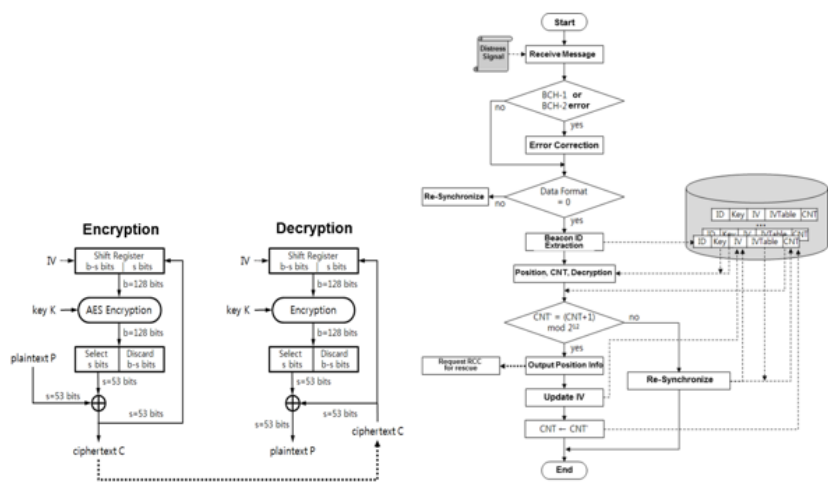

Fig. 7. Encryption by 128 bits AES/CFB \& Key management 


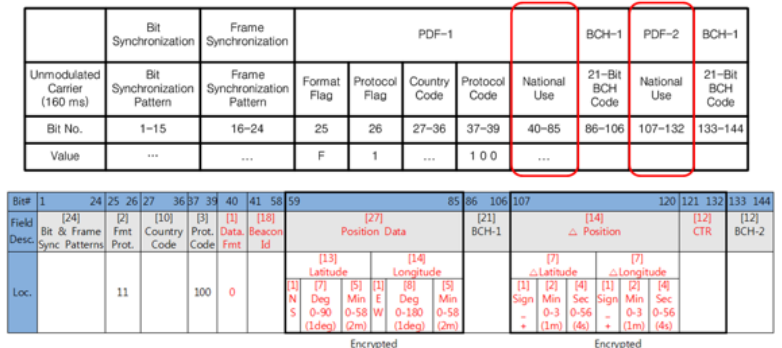

Fig. 8. National User Protocol message format and data fields from COSPAS SARSAT message structure[7].

LTE link provides its own security mechanism and additional security mechanism by using AES 256 bits for LTE was used. Encryption algorithm with IPSec protocol was applied including LTE user terminal control through return link, MCC encryption message processing, and encryption key management through return link.

Additionally, COSPAS SARSAT link based on USRP in RF was utilized to check its feasibility as shown in Fig. 9.

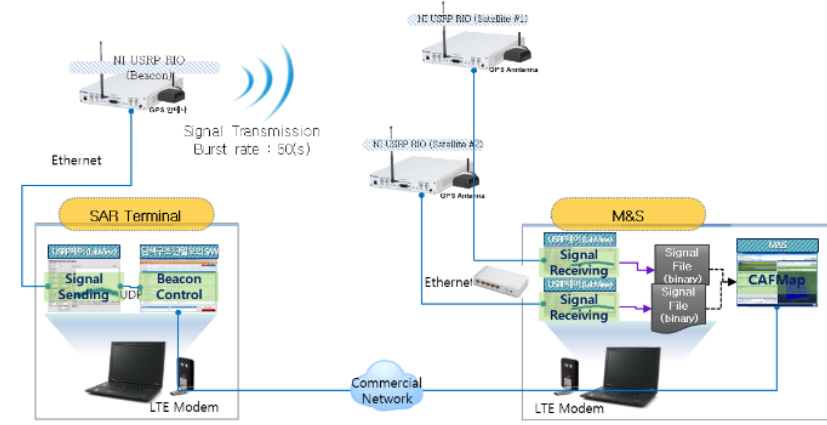

Fig. 9. USRP based CCOSPAS SARSAT M\&S.

Also, signal by taking into account dynamics of satellite and relative motion of the satellite [8] were applied to the hybrid system as shown in Fig. 10. We, also, applied new COSPAS SARSAT signal scheme in spread spectrum was applied to the system.

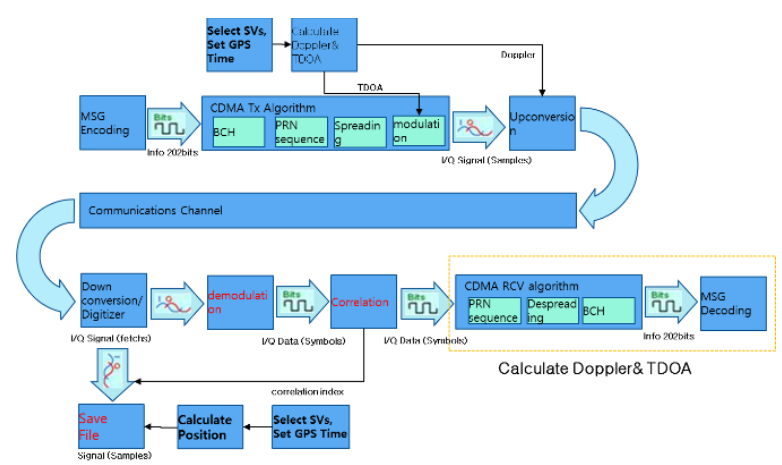

Fig. 10. Dynamic CCOSPAS SARSAT signals

\section{Conclusions}

Results of the study on Search and Rescue System for Military and Civil use by using COSPAS SARSAT and Terrestrial Communication Network was described. The study has been made to show the feasibility of the system under consideration of prompt implementation. System was designed by considering less burden to conventional COSPAS SARSAT, employ encryption for security and privacy, alternative positioning with distress signal itself, and RF level signal feasibility check. Based on the study, commercialization and mass production of the beacon including system is proposed as future work.

This work has been supported by National GNSS Research Center program of Defence Acquisition Program Administration and Agency for Defence Development.

\section{References}

1. COSPAS SARSAT(2017), https://www.cospassarsat.int/en/system-overview/cospas-sarsat-system

2. Kim, J. H., Lee, S. U., Kim, J. H., Ahn, W. G. 2014, Development Status of Military Search and Rescue System M\&S Software, The Journal of Korea Society of Communication and Space Technology, Vol 9 No $3,121-126$

3. Sanguk Lee, I. C. Jeong, Jaehyun Kim, and Woo-Geun Ahn, " Security enhanced Search and Rescue System by using LTE and COSPAS SARSAT, " 11th Annual Baska GNSS Conference, Baska, Kirk Island, Croatia. 5. 7-9, 2017

4. FIPS(2001), FIPS-197, Advanced encryption standard (AES).

5. IEEE(2007), "Standard for cryptographic protection of data on block-oriented storage services," IEEE P1619/D16.

6. Stallings, W. (2011), Cryptography and Network Security, 5th Ed., Pearson.

7. COSPAS SARSAT(2012A), JC-26/Inf.27, SAR/GALILEO Return Link System Message Specification

8. Jeong, I. C., Kim, D. W., Ahn, W. G., \& Lee, S. U., 2017, Development of Search and Rescue System with Dynamic Model by RF Signal Based LTE, Journal of Satellite, information and Communications, Vol 12 No.4, 120-124 